\title{
Original
}

\section{Mortalidad por causas accidentales en niños menores de 5 años en el Hospital Nacional de Niños "Dr. Carlos Sáenz Herrera", 2002-2004}

\author{
(Mortality due to accidental causes in children younger \\ than 5 years of age at the Hospital Nacional de Niños \\ " Dr. Carlos Sáenz Herrera", 2002-2004)
}

Adriana Benavides-Lara', Marco Vargas-Salas²

\section{Resumen}

I. Centro Nacional de Referencia de Enfermedades Congénitas. Instituto Costarricense de Investigación y Enseñanza en NutriciónySalud(INCIENSA)

2. Unidad de Trauma y Unidad de Emergencias Quirúrgicas, Hospital Nacional de Niños. Abreviaturas: AVPP, años de vida potencialmente perdidos; CCP, Centro Centroamericano de Población; CIE-10, Clasificación Internacional de Enfermedades y Problemas Relacionados con la Salud, Décima de Revisión, 1995; HNN, Hospital Nacional de Niños "Dr. Carlos Sáenz Herrera"; INEC, Instituto Nacional de Estadística y Censos; OMS, Organización Mundial de la Salud. Correspondencia: Adriana Benavides Lara. Instituto Costarricense de Investigación y Enseñanza en Nutrición y Salud, INCIENSA. Apartado 4-2250, Tres Ríos, Cartago, Costa Rica. Email:abenavides@ inciensa.sa.cr
Las lesiones traumáticas por causas externas corresponden en Costa Rica a la segunda causa de muerte en niños menores de 5 años, con una tasa para 2004 de 9.23 x 100000 habitantes, y la primera causa de años de vida potencialmente perdidos $(37 \%)$ para esta edad. El objetivo de esta investigación fue evaluar el impacto de este tipo de lesiones como causa de muerte en niños menores de 5 años, atendidos en el Hospital Nacional de Niños (HNN), y caracterizar esta población.

Materiales y métodos: Se realizó un estudio caso control pareado, donde se incluyeron todos los niños menores de 5 años egresados del HNN desde diciembre de 2001 a diciembre de 2004, con diagnóstico de lesión no intencional por causas externas. Los casos los constituyeron los niños que egresaron fallecidos y para cada uno se eligió al azar un control de la misma edad y sexo que no hubiera fallecido por causa de la lesión traumática.

Resultados: Hubo un total de 36 casos distribuidos igualmente por sexo. Las principales causas de muerte resultaron, en orden de importancia: los accidentes de tránsito y los ahogamientos. La mayoría de los casos provinieron de la provincia de San José. No se encontraron diferencias estadísticamente significativas en la mayoría de variables estudiadas entre casos y controles. La mayoría de muertes ocurrieron durante los meses de verano y típicos de vacaciones escolares; además, el 35\% de estas ocurrieron durante el fin de semana. La mayoría de los niños ingresó al hospital durante el segundo turno $(2 \mathrm{pm}-10 \mathrm{pm})$ y la gran mayoría de las lesiones (64\%) ocurrieron en la casa $(\mathrm{p}<0.05)$. La no supervisión figuró, dentro del estudio, como un factor de riesgo de ocurrencia de una lesión no intencional $(\mathrm{p}<0.05)$.

Conclusiones: El registro de este tipo de lesiones es aún muy deficiente en Costa Rica, lo cual dificulta la realización de un estudio analítico sobre prevalencia y factores de riesgo de lesiones no intencionales por causas externas.

Descriptores: Lesiones traumáticas, causas externas, accidentes de tránsito, trauma en niños.

\section{Abstract}

In Costa Rica unintentional injuries are the second cause of mortality in children under 5 years of age, with a ratio of 9.23 per 100000 inhabitants for the year 2004 . They are also the leading 
ISSN 0001-6002/2008/50/1/22-28 Acta Médica Costarricense, (C2007 Colegio de Médicos y Cirujanos cause of loss of productive life years (37\%) for this age group. The objetive of this study was to determine the impact of this kind of injuries over the mortality of children under 5 years of age seen at the Hospital Nacional de Niños (HNN) and to characterize this population.

Methods: We performed a case-control study pooling data on chilren aged 0-5 years who had been discharged from HNN with the diagnosis of unintentional injury from december 2001 to december 2004. All children who died from these injuries during this period constituded the cases and for each one we choose a control with the same age, sex and diagnosis discharged alive for this cause.

Results: There were 36 cases (deaths from unintentional injuries) $50 \%$ of each sex. Motor vehicle related injuries and drownings were the leading causes of death. Most of them were from San José. There was no statatistical difference betwen cases and the controls in the mayority of variables. Most of the deaths were during the summer and vacational months, beside $35 \%$ of them ocurred on weekends. Most of the cases were admitted during the second shift $(2 \mathrm{pm}$ $10 \mathrm{pm})$ moreover the mayority of injuries $(64 \%)$ took place at home $(\mathrm{p}<0.05)$. No supervision of children resulted to be risk factor for an unintentional injury ocurrence $(\mathrm{p}<0.05)$, but not for mortality.

Conclusion: The Injury Registry in Costa Rica is still very deficient, which makes difficult to carry out analitycal sutdies of prevalence of risk factors of unintentional injuries.

Key words: traumatic lesions, external causes, traffic accidents, children trauma

Recibido: 14 de junio de 2006

Aceptado: 25 de septiembre de 2007
Según la Organización Mundial de la Salud, el trauma se define como un daño intencional o no intencional, causado al organismo por su brusca exposición a fuentes o concentraciones de energía (mecánica, química, térmica o radiante) que sobrepasan su margen de tolerancia, o factores que interfieren con intercambios de energía en el organismo (deprivación de elementos esenciales para la vida como calor, comida u oxígeno). Es la tercera causa de muerte en el mundo para todos los grupos de edad, y la primera causa mundial de años de vida potencialmente perdidos (AVPP). ${ }^{1,2}$ Alrededor del mundo la mortalidad por lesiones traumáticas presenta ciertas tendencias y características según el género, el grupo etario, el nivel socioeconómico y el mecanismo de lesión, siendo los accidentes de transporte (de tránsito o de vehículos automotores) los que cobran más vidas. ${ }^{3-8}$ Para gran parte de las causas externas, el género masculino presenta la mayor tasa de mortalidad. ${ }^{2}$ Globalmente, el $90 \%$ de las muertes traumáticas ocurren en países de mediano y bajo nivel económico. ${ }^{2}$ Los niños menores de 5 años (con 2 subgrupos: infantes y de 1-4 años) son, para efectos del estudio, el área de interés.

En Europa, las lesiones traumáticas representan el 30\% de la causa de mortalidad en niños. El patrón de las lesiones es similar al observado en los demás continentes y los principales mecanismos de lesiones no intencionales son, en su orden: accidentes de transporte, ahogamiento por sumersión, fuego y quemaduras, caídas $\mathrm{y}$ envenenamientos. ${ }^{2,9}$

En América las lesiones traumáticas representan el 5\% de todas las defunciones registradas en niños menores de 5 años, y la segunda causa de muerte, después de las enfermedades transmisibles para la misma edad. ${ }^{1}$

Según datos de la Organización Panamericana de la Salud, en Costa Rica, hasta antes del año 2000, las lesiones traumáticas no intencionales eran la segunda causa de muerte después de las enfermedades transmisibles, para niños menores de 5 años y mayores de 1 año. Sin embargo, en el último quinquenio han pasado a ser la segunda causa de muerte después de las malformaciones congénitas. En los niños menores de 1 año, los accidentes son la tercera causa de mortalidad después de las entidades originadas en el periodo perinatal y las enfermedades congénitas. ${ }^{1} \mathrm{Al}$ igual que en las demás partes del mundo, el género masculino supera desde el primer año la mortalidad y la morbilidad por esta causa. Pero, las tasas de mortalidad por lesiones en niños y niñas llevan la misma tendencia a lo largo de los años.

Este tipo de lesiones son, además, la principal causa de AVPP, ocasionando el $37 \%$ de ellos, seguidos de los tumores con un $23 \%$ y las enfermedades del aparato circulatorio con un $19 \% .^{10}$

El HNN creó, en diciembre de 2001, la Unidad de Trauma y Unidad de Emergencias Quirúrgicas, que se ha convertido desde hace un poco más de 3 años, en el centro de referencia nacional para los niños lesionados por traumatismos. Atiende a todos los niños que han sufrido este tipo de evento, que no puedan ser manejados en hospitales regionales, y a la mayoría de los niños que sufren accidentes con lesiones severas. 
En Costa Rica no existe un estudio poblacional en donde se trate de caracterizar la muerte por causas externas en los niños, sino más bien estudios de causas externas específicas de lesiones traumáticas. ${ }^{11-14}$ No se cuenta con un sistema uniforme de registro para estas muertes, las cuales, según el centro, se clasifican de diferente manera. Esto dificulta que se pueda hacer un estudio poblacional serio al respecto.

El objetivo de esta investigación fue definir el impacto de las lesiones no intencionales por agentes externos como causa de muerte en niños menores de 5 años, admitidos al Hospital Nacional de Niños "Dr. Carlos Sáenz Herrera" (HNN), y caracterizar esta población, por ser el centro de referencia de trauma pediátrico en el nivel nacional.

\section{Materiales y métodos}

Se realizó un estudio caso-control pareado. La población incluyó a todos los niños menores de 5 años egresados del $\mathrm{HNN}$ del $1^{\circ}$ de diciembre de 2000 el $1^{\circ}$ de diciembre de 2004, cuyo diagnóstico de egreso pudiera ser clasificado según la CIE-10, como lesiones no intencionales por causas externas (CIE 10 entre V00 y X99).

De esta población, los casos fueron todos los niños menores de 5 años que egresaron fallecidos a consecuencia de lesiones traumáticas no intencionales causadas por agentes externos, en el período citado. Para cada caso, se eligió al azar un control entre los niños egresados vivos por el mismo motivo durante 2003, agrupándolos por sexo, edad y causa de lesión.

Las causas de lesión se clasificaron de acuerdo con la décima Clasificación Internacional de las Enfermedades (CIE-10). Estas abarcan el capítulo XX de dicha clasificación, desde la V00 hasta la Y 98, según su codificación. Se incluyeron en el estudio solamente aquellas donde no hubo intencionalidad de causar la lesión, por lo tanto, se excluyeron todas las causas autoinflingidas (X60-X84), agresiones (X85-Y09), eventos clasificados como de intención no determinada, es decir, aquellos donde no se anotó nada acerca de la intencionalidad y que no están en investigación (Y10-Y34), y otras relacionadas con eventos de guerra, mala praxis y factores vinculados con causas externas (Y40-Y98).

Por ser un estudio no intervencionista, en donde se mantiene en todo momento la confidencialidad de la identidad de los sujetos sometidos a descripción, no se requirió de consentimiento informado y fue previamente avalado por el comité de Bioética del Hospital Nacional de Niños (UBIHNN).

Como fuentes de información se utilizaron: los registros de egresos de la unidad de estadística del HNN, los expedientes clínicos (microfilmados o de papel) y las actas de defunción.
Cuadro 1: Sexo, nacionalidad y procedencia

\begin{tabular}{|lccccccc|}
\hline & \multicolumn{3}{c}{ Casos } & \multicolumn{3}{c|}{ Controles } \\
\hline & $\mathrm{N}$ & $\%$ & IC95\% & $\mathrm{N}$ & $\%$ & IC95\% & $\mathrm{p}$ \\
\hline Sexo & & & & & & & \\
\hline Masculino & 18 & 50.0 & & 18 & 50.0 & & \\
Femenino & 18 & 50.0 & & 18 & 50.0 & & \\
\hline Nacionalidad & & & & & & & \\
\hline Costarricense & 34 & 94.4 & $81.3-99.3$ & 35 & 97.2 & $85.5-99.9$ & \\
Nicaragüense & 0 & 0.0 & -- & 1 & 2.8 & $0.1-14.5$ & \\
Otro & 2 & 5.6 & 07.18 .7 & 0 & 0.0 & -- & 0.22 \\
\hline Provincia & & & & & & & \\
\hline San José & 16 & 44.4 & $27.9-61.9$ & 19 & 52.8 & $35.5-69.6$ & \\
Alajuela & 5 & 13.9 & $4.7-29.5$ & 5 & 13.9 & $4.7-29.5$ & \\
Cartago & 4 & 11.1 & $3.1-26.1$ & 3 & 8.3 & $1.8-22.5$ & \\
Heredia & 3 & 8.3 & $3.1-26.1$ & 1 & 2.8 & $0.1-14.5$ & \\
Guanacaste & 1 & 2.8 & $0.1-14.5$ & 3 & 8.3 & $1.8-22.5$ & 0.8 \\
Puntarenas & 4 & 11.1 & $3.1-26.1$ & 2 & 5.6 & $0.7-18.7$ & \\
Limón & 3 & 8.3 & $1.8-22.5$ & 3 & 8.3 & $1.8-22.5$ & \\
\hline
\end{tabular}

\begin{tabular}{|lccccccc|}
\hline \multicolumn{5}{c}{ Cuadro 2. Sitio del evento } \\
\hline \multicolumn{7}{c}{ Caso } & \multicolumn{3}{c|}{ Control } \\
\hline \multicolumn{1}{|c}{ Sitio accidente } & $\mathrm{n}$ & $\%$ & $\mathrm{IC} 95 \%$ & $\mathrm{n}$ & $\%$ & $\mathrm{IC} 95 \%$ \\
\hline Casa materna & 16 & 44.44 & $42.5-82.0$ & 15 & 44.1 & $27.2-62.1$ \\
Zona de recreación & 3 & 8.3 & $1.8-22.5$ & 6 & 17.6 & $6.8-34.5$ \\
Otro & 2 & 5.6 & $0.7-18.7$ & 1 & 2.9 & $0.1-15.3$ \\
No especificado & 4 & 11.1 & $3.1-26.1$ & 1 & 2.9 & $0.1-15.3$ \\
Accidentes de transporte & & & & & & \\
Accidente tránsito (vía & 9 & 25.0 & $12.1-42.2$ & 11 & 32.4 & $17.4-50.5$ \\
pública) & & & & & & \\
Accidente no de tránsito & 1 & 2.8 & $0.1-14.5$ & 0 & 0.0 & $0.0-10.3$ \\
No especificado & 1 & 2.8 & $0.1-14.5$ & 0 & 0.0 & $0.0-10.3$ \\
\hline
\end{tabular}

Los resultados se analizaron con el programa Epi-Info ${ }^{\circledR}$, versión 3.3.2 de 2005. Se analizaron frecuencias y test de significancia estadística con un valor de $\mathrm{p}<0.05$, con intervalos de confianza superiores al 95\% (T-student y Chi- cuadrado).

\section{Resultados}

Durante los 4 años del estudio se encontraron 36 casos de niños menores de 5 años fallecidos, cuya muerte fue por una lesión por causa externa. En el Cuadro 1 se muestran sexo, nacionalidad y procedencia de la población de estudio. Al comparar la distribución de las variables de nacionalidad y provincia de residencia entre los casos y los controles, se 
evidenció que no existe diferencia en la distribución entre ambos grupos ( $\mathrm{p}>0.05$ ). La edad entre los casos varió de 16 días a 4 años- 11 meses, con un promedio en meses al momento de la atención, de 29.3 meses (IC95\% 23.1-35.6). Del total de 36 casos hubo 8 menores de 1 año (22\%) y 28 de 1 a 4 años (78\%). Los días de estancia hospitalaria fueron en promedio 3.4 y los controles, 3.9 días, sin que se demostrara diferencias significativas entre ambos $(\mathrm{p}=0.73)$.

Los meses prevalentes fueron los de febrero, abril y mayo, con un 13.9\%(IC95\% 4.7-29.5) para cada mes, en el grupo de los casos, y febrero con un 16.7\% (IC95\%6.432.8 ), seguido por junio con un $13.9 \%$ (IC95\% 4.7-29.5) y diciembre en igual proporción. La diferencia entre meses aislados no fue estadísticamente significativa $(\mathrm{p}=0.27)$.

Los días sábado, domingo y lunes fueron los más frecuentes para la ocurrencia de lesiones accidentales, tanto en los casos como en los controles $(\mathrm{p}=0.74)$.

\begin{tabular}{|lllllllll|}
\hline \multicolumn{3}{|c|}{ Cuadro 3. Supervisión durante el evento } \\
\hline \multirow{2}{*}{ Supervisión } & \multicolumn{3}{c}{ Casos } & \multicolumn{3}{c|}{ Controles } & \\
\cline { 2 - 7 } & $\mathrm{N}$ & $\%$ & $\mathrm{IC} 95 \%$ & $\mathrm{~N}$ & $\%$ & $\mathrm{IC} 95 \%$ & $\mathrm{p}$ \\
No & 27 & 75 & $57,8-87,9$ & 18 & 50 & $32,9-67,1$ & \\
No se sabe & 3 & 8,3 & $1,8-22,5$ & 16 & 16,7 & $6,4-32,8$ & 0,09 \\
Sí & 6 & 16,7 & $6,4-32,8$ & 12 & 33 & $18,6-51,0$ & \\
\hline
\end{tabular}

Se evidenció además que el $80 \%$ de los accidentados, tanto en casos como en controles, recibieron asistencia médica antes de su ingreso al HNN.

El 13.9\% (IC95\% 4.7-29.5) de los casos murieron sin poder descartar intencionalidad (agresión) en su muerte; todos ellos eran menores de 18 meses. En el $2.8 \%$ del grupo

\begin{tabular}{|c|c|c|c|c|c|c|}
\hline & & Cuadro 4: & Mecanismo & e lesión & & \\
\hline \multirow[t]{2}{*}{ Característica* } & \multicolumn{6}{|c|}{ Tipo de causa externa de lesión } \\
\hline & Sumersión & Caídas & Quemaduras & Golpes & Transporte & Asfixia \\
\hline Total (\%) & $11(31)$ & $6(17)$ & $4(11)$ & $2(6)$ & $11(31 \%)$ & $2(6)$ \\
\hline $\begin{array}{l}\text { Menores de } 1 \text { año } \\
(\%)\end{array}$ & $\begin{array}{c}1 \\
9 \%\end{array}$ & $\begin{array}{c}2 \\
33 \%\end{array}$ & $\begin{array}{c}1 \\
25 \%\end{array}$ & $\begin{array}{c}1 \\
50 \%\end{array}$ & $\begin{array}{c}2 \\
18 \%\end{array}$ & $\begin{array}{c}1 \\
50 \%\end{array}$ \\
\hline $\begin{array}{l}\text { De } 1 \text { a } 4 \text { años } \\
(\%)\end{array}$ & $\begin{array}{c}10 \\
91 \%\end{array}$ & $\begin{array}{c}4 \\
67 \%\end{array}$ & $\begin{array}{c}3 \\
75 \%\end{array}$ & $\begin{array}{c}1 \\
50 \%\end{array}$ & $\begin{array}{c}9 \\
69 \%\end{array}$ & $\begin{array}{c}1 \\
50 \%\end{array}$ \\
\hline $\begin{array}{l}\text { Edad promedio } \\
\text { en años }\end{array}$ & 2.6 & 1.1 & 17.8 & Menores de 1 & 2.2 & Menores de 2 \\
\hline $\begin{array}{l}\text { Sexo } \\
\text { (n) }\end{array}$ & $\begin{array}{l}\text { Femenino (6) } \\
\text { Masculino (5) }\end{array}$ & $\begin{array}{l}\text { Femenino (4) } \\
\text { Masculino (2) }\end{array}$ & $\begin{array}{l}\text { Femenino (2) } \\
\text { Masculino (2) }\end{array}$ & Femenino (2) & $\begin{array}{l}\text { Femenino (3) } \\
\text { Masculino (8) }\end{array}$ & $\begin{array}{l}\text { Femenino (1) } \\
\text { Masculino (1) }\end{array}$ \\
\hline $\begin{array}{l}\text { Provincia con más } \\
\text { casos }(n)\end{array}$ & San José (5) & San José (3) & San José (2) & Ninguna & San José (5) & Ninguna \\
\hline $\begin{array}{l}\text { Estancia hospitalaria } \\
\text { promedio días }\end{array}$ & 6,3 & 2 & 0.25 & 1 & 2.18 & 0.5 \\
\hline Supervisión (n) & No (11) & No (4) & No (4) & No (2) & No (8) & No (1) \\
\hline $\begin{array}{l}\text { Sitio del suceso } \\
\text { (n) }\end{array}$ & $\begin{array}{c}\text { Casa (7) } \\
\text { Recreativo (3) }\end{array}$ & Casa (5) & Casa (3) & Casa (2) & $\begin{array}{l}\text { Vía pública } \\
\text { (9) }\end{array}$ & Casa (2) \\
\hline Mes más frecuente & $\begin{array}{l}\text { Agosto } \\
\text { Noviembre }\end{array}$ & $\begin{array}{l}\text { Mayo } \\
\text { Agosto }\end{array}$ & Ninguno & $\begin{array}{l}\text { Febrero } \\
\text { Abril }\end{array}$ & $\begin{array}{l}\text { Diciembre } \\
\text { Marzo, Julio }\end{array}$ & $\begin{array}{l}\text { Octubre } \\
\text { Enero }\end{array}$ \\
\hline Día más frecuente & $\begin{array}{l}\text { Fin de semana } \\
\text { (7) }\end{array}$ & $\begin{array}{l}\text { Fin de } \\
\text { semana (4) }\end{array}$ & Ninguno & $\begin{array}{l}\text { Entre semana } \\
\text { (2) }\end{array}$ & $\begin{array}{l}\text { Entre semana } \\
\qquad(8)\end{array}$ & $\begin{array}{c}\text { Entre semana } \\
\text { (2) }\end{array}$ \\
\hline Supervisión (n) & No (11) & No (4) & No (4) & No (2) & No (8) & No (1) \\
\hline
\end{tabular}


control hubo sospecha de síndrome de niño agredido (IC95\% 0.1-14.5). Las diferencias, aunque notables, no resultaron estadísticamente significativas $(\mathrm{p}=0.54)$. La mayoría de los pacientes ingresaron en el segundo turno, que va de 2 a 10 pm, tanto en el grupo de casos (41.7\%) como en el de controles $(44.4 \%)$, sin haber diferencia estadísticamente significativa entre ellos $(\mathrm{p}=0.959)$. Al determinar la hora de ocurrencia de los hechos, el periodo de mayor frecuencia fue entre la $1 \mathrm{pm}$ y las $5 \mathrm{pm}$ para el grupo de casos (36.1\%), y el período comprendido entre las 6 am y las $12 \mathrm{md}$ para el grupo de control(47.1\%), sinser la diferencia estadísticamente significativa $(\mathrm{p}=0.23)$.

Tanto en el grupo de casos como en el grupo de controles, la casa materna fue el sitio más frecuente del evento (Cuadro 2), y no hubo diferencias significativas entre los grupos. En cuanto a la existencia de supervisión durante la ocurrencia de lesión, hay un claro predominio de no supervisión (Cuadro $3)$, sin que haya diferencia estadísticamente significativa entre casos y controles.

La edad materna no se pudo determinar en el $66.7 \%$ de los casos (IC95\% 49.0-81.4) y las madres mayores de edad fueron un 33.3\% (IC95\%18.6-51.0) en este grupo. En el de control hubo un 75.0\% (IC95\%57.8-87.9) de madres mayores de edad. La diferencia entre casos y controles fue estadísticamente significativa $(\mathrm{p}<0.001)$. El Cuadro 4 resume de las principales características para cada mecanismo de lesión en los niños que fallecieron por estas causas.

\section{Discusión}

En el HNN mueren, por lesiones por causas externas no intencionales, un promedio de 9 niños menores de 5 años, lo que corresponde a un total del 3\% de las muertes dentro de ese rango de edad en el Hospital. Separando por grupos de edad a los infantes ( $<1$ año), los cuales mueren generalmente de causas perinatales o malformaciones congénitas, las muertes accidentales aportan un $0,8 \%$ al total de las muertes de niños de esa edad. Por otro lado, la contribución porcentual de las muertes accidentales en la mortalidad total de niños de 1 a 4 años en el HNN de 2001 a 2004 fue del $13,9 \%$. Estos datos van de acuerdo con las estadísticas nacionales, donde los accidentes son la principal causa de muerte en niños de 1 a 4 años, siendo la tercera causa de muerte para los niños menores de 1 año, después de las causas perinatales y las malformaciones congénitas. Además, según datos del Instituto Nacional de Estadística y Censos (INEC), la tasa de mortalidad por lesiones no intencionales en niños menores de 5 años ha tendido a aumentar con los años de un 5.8 por 100000 para 2000, a un 9.23 por 100000 para 2004. Este aumento es básicamente a expensas del grupo de niños menores de 1 año, cuya tasa aumentó de 5 a 13,04 x100000, mientras que el grupo de 1 a 4 años aumentó de 4,0 a 8,30 x 100000 para los años 2000 y 2004, respectivamente.

Con respecto al sexo, el aporte porcentual de cada uno a los casos en este estudio fue del $50 \%$, lo cual no refleja la realidad nacional. Según datos del INEC para el último quinquenio 2000-2004, en niños menores de 1 año el aporte porcentual por sexo no fue estadísticamente significativo y el $54 \%$ de las muertes accidentales fueron en mujeres. Sin embargo, para niños de 1 a 4 años, el sexo masculino supera al femenino, con tasas de $1.32 \times 10000$ (IC 95\% 1,05-1,63) versus $0.35 \times 10000$ (IC 95\% 0,00-0,98), diferencia que se acentúa haciéndose estadísticamente más significativa con la edad. Al igual que en el nivel nacional, el estudio evidenció que en niños menores de un año, la mayoría de los casos los conforman mujeres $(62 \%)$. No obstante, se necesita un estudio que abarque una población mayor, para determinar si el sexo representa un factor de riesgo para sufrir accidentes y para morir de estas causas en niños menores de 5 años en Costa Rica. Mundialmente, varios estudios han demostrado que el género masculino presenta la mayor tasa de mortalidad y prevalencia de lesiones por causas externas. ${ }^{2,5-9}$

La mayoría de casos y de controles provinieron de San José, debido a que el HNN se encuentra en esta provincia y sus pacientes principalmente provienen de esta, de Alajuela y de Heredia. Por otro lado, ya que muchos de los niños accidentados del país viven en provincias alejadas, con otros hospitales centrales (Guanacaste y Limón), estos acuden primero a dichos centros y muchos fallecen en ellos. Incluso, según datos del Centro Centroamericano de Población de la Universidad de Costa Rica (CCP), para el quinquenio 19982002, cerca del $74 \%$ de los niños menores de 5 años fallecidos por causas externas, mueren sin asistencia médica, y del $55 \%$ al $60 \%$ no mueren en un centro de salud (hospital, clínica o EBAIS), sino que lo hacen en la casa, en la escena o en otros lugares. En contraparte, la gran mayoría de los niños ingresados al $\mathrm{HNN}$ vienen en ambulancia y con asistencia médica, lo cual sugiere, que a este hospital llegan los accidentados que forman parte de la minoría del $26 \%$ que sí reciben asistencia médica en el país y que después del accidente tiene oportunidad, por acceso a servicios de salud o por cercanía con el hospital, de llegar a ser atendidos en este centro. El resto de la población, que es el grueso, se escapa de este estudio, por lo cual, se hace necesario, para poder caracterizar las lesiones por causas externas en Costa Rica, un estudio multicéntrico que abarque al menos los principales centros hospitalarios de urgencias del país.

El promedio de estancia de casos y controles fue de 3,6 días, bastante menor al reportado en estudios de la Unidad de trauma del HNN para trauma mayor severo, con 10,8 días promedio. ${ }^{13}$ Sin embargo, tales estudios incluyeron niños hasta de 14 años, y este solo a menores de 5 años. 
La mayor cantidad de muertes accidentales sucedió en los meses de vacaciones de verano, (diciembre a febrero), abril (semana santa) y junio (medio año). Este dato coincide con lo anotado mundialmente, ${ }^{2,7,15,16}$ exceptuando los accidentes por fuego, humo y quemaduras, que son prevalentes durante los meses de invierno, ${ }^{16-18} \mathrm{y}$ las muertes por asfixia y sofocación, que no presentan predilección por temporadas. ${ }^{2,16}$ Los días de fin de semana son los de mayor prevalencia de lesiones por causas externas, tal como lo menciona la bibliografía mundial, ,,12,17-19 $\sin$ haber diferencia estadística entre casos y controles. Por lo tanto, el día de la semana es más bien un factor de riesgo para la ocurrencia de estos accidentes.

En relación con la hora de ingreso al hospital, no hay estudios que hayan evaluado esta variable. Si la mayoría de lesiones no intencionales suceden en el hogar de estos niños, es de esperar que ingresen al hospital una vez que hayan llegado a sus casas de centros de cuidos diurnos o guarderías.

Excluyendo los accidentes de transporte, de los cuales el $82 \%$ sucedieron en la vía pública (accidentes de tránsito), en la casa materna ocurrió un $64 \%$ de los eventos de los casos, siendo esta diferencia estadísticamente significativa, tanto para los casos como para los controles $(p<0.05)$. La información está apoyada por estudios mundiales, en los cuales la casa materna sigue siendo el lugar más peligroso para que los niños sufran accidentes, en comparación con guarderías, centros de estudio y otros lugares, sin importar el tiempo de exposición en cada sitio. $5,7,17-22$

La supervisión durante el evento de lesión no figura como un factor de riesgo para morir por este tipo de lesiones en niños menores de 5 años, ya que ambos, casos y controles, se dieron en su mayoría en circunstancias de no vigilancia por un adulto, sin embargo, la diferencia entre niños supervisados y no supervisados de ambos grupos es estadísticamente significativa ( $\mathrm{p}<0.05)$, lo cual lo convierte en un factor de riesgo para sufrir la lesión, tal y como ha sido demostrado por numerosos estudios internacionales. ${ }^{3-5,8,23}$

Según el INEC, para el período 2000-2004, las principales causas de muerte por accidentes las ocupan, en orden: los accidentes de transporte, los ahogamientos por sumersión y las quemaduras en el grupo etario de 1 a 4 años, y la sofocación y asfixia, los accidentes de transporte y las caídas en menores de 1 año. Comparando este dato con los casos encontrados en el HNN, se demuestra que el mayor porcentaje de muertes por accidentes fue originado por los ahogamientos por sumersión, seguidos de los accidentes de transporte. Llama la atención que entre los casos del HNN las caídas ocupan el tercer lugar como causa de muerte por accidentes, con un total de 6 casos, sin embargo, en el INEC solo reportan en el mismo lapso 3 muertes por esa causa, en menores de 5 años en el nivel nacional. Esto muestra una vez más, la discordancia entre diferentes registros, y el subregistro que puede haber al respecto en Costa Rica, al no existir un método de codificación y clasificación que se enseñe tanto a médicos como a personal de registros médicos. Por otro lado, internacionalmente, las caídas ocupan entre el tercero y el cuarto puestos como causa de muertes por lesiones accidentales, lo cual concuerda con los datos obtenidos en el estudio. Los otros mecanismos como las quemaduras, la sofocación o asfixia y los golpes, son los siguientes en frecuencia, tanto en el nivel nacional como en el estudio.

Comparando el ámbito nacional con el presente estudio en el HNN, se puede evidenciar que ambos tienen una distribución similar por grupo de edad. En ambos, la mayoría de los niños menores de 1 año mueren por caídas y sofocación y asfixia, pero sí hubo diferencia en los niños fallecidos por golpes (o exposición a fuerzas mecánicas inanimadas y animadas), donde en el nivel nacional solo el $11 \%$ corresponden a menores de 1 año; en cambio, en el HNN el $50 \%$ de los niños muertos por esa causa fueron infantes. Con respecto a los ahogamientos y accidentes de tránsito, tanto en el nivel nacional como en el estudio el mayor porcentaje se presentó en niños de 1 a 4 años, al igual que en las muertes por quemaduras.

La edad de las madres de los niños incluidos en este estudio no se pudo determinar en el $66,7 \%$ de los casos, lo cual revela la necesidad de agudizar el registro y caracterización de estas muertes, ya que mundialmente se ha demostrado que existe mayor riesgo de mortalidad y morbilidad por lesiones por causas externas no intencionales, en niños de madres menores de 20 años., ${ }^{3,4,8,15-17,24-26}$ Estos mismos estudios revelan que el bajo nivel socioeconómico, así como la paridad y el estado civil de la madre son factores de riesgo independientes de mortalidad por lesiones accidentales.

Muchos datos determinantes en el riesgo de mortalidad por lesiones no intencionales no son registrados en el expediente clínico y son imprescindibles para realizar un estudio epidemiológico que determine causalidad de algunos de estos con el fin de implementar políticas de prevención dirigidas al ambiente nacional. Debe también efectuarse con premura un estudio de los comportamientos más susceptibles de ser regulados jurídicamente para prevenir este tipo de lesiones.

No existe en el país un registro unificado de estas lesiones, lo que dificulta que se pueda desarrollar un sistema de vigilancia estructurado, con estándares de clasificación y codificación.

Como principal conclusión queda entonces una gran interrogante: ¿cuántas de las muertes por lesiones "accidentales" en los niños costarricenses son prevenibles, legislativamente, médicamente o supervisando a los niños con mayor conciencia de los riesgos existentes?. Se sabe que estudios internacionales, de países que cuentan ya con un buen registro de estos eventos y de sus factores de riesgo, han encontrado que más del $90 \%$ de estas muertes pudieron haberse prevenido. 


\section{Referencias}

1. Organización Panamericana de la Salud. Estadísticas de Salud de las Américas. 4ta edición. Organización Panamericana de la Salud 1998; 29-62

2. Organización Mundial de la Salud. Defunciones por accidentes de tránsito en países seleccionados de las Américas, 1985-2001. OMS Boletín Epidemiológico 2004; 25: 2-5.

3. Scholer S, Hickson G and Ray W. Sociodemographic factors indentify US infants at high risk of injury mortality. Pediatrics 1999; 103: 11831188.

4. Scholer S, Mitchel,E and Ray, W. Predictors of injury mortality in early childhood. Pediatrics 1997; 100:342-347.

5. Wayne R. Prevention or Household and Recreational injuries in children. In: Canadian Task Force on the Periodic Health Examination. Canadian guide to Clinical Preventive Health. Ottawa: Health Canada; 1994: 306-317.

6. Guyer B, Ellers, B. Childhood injuries en the United States: mortality, morbidity and cost. Am J Dis Child 1990; 144: 649-752.

7. Waller AE, Bake SP, Szocka A. Childhood injury deaths: national analysis and geographic variations. Am J Public Health 1989; 79: 310-315.

8. Peclet MH, Newman KD, Eichelberger MR, Gotschall K, Guzzetta P, Anderson K et al. Patterns of injury in children. J Pediatr Surg 1990; 25: 85-91.

9. Morison A. Unintentional childhood injury mortality in Europe 198493: a report from the EURORISC Working Group. Inj Prev 1999; 5: 171-176.

10. Caja Costarricense de Seguro Social. Plan de atención a la salud de las personas: PASP 2001-2006. Edición 2001. San José: Caja Costarricense del Seguro Social; 2001. 39-47.

11. Lobo F, Vernon M, Quesada A, González M. Accidentes de tránsito en niños: aspectos médicos y epidemiológicos. Act Méd Costarric 1991; 34: 65-69.

12. Quirós D. Intoxicaciones atendidas en el Hospital Nacional de Niños durante 1990-1996. Acta Pediát Cost 1998; 12: 28-31.

13. Vargas M, Moraga M, Chacón F, Elizondo I. Trauma mayor severo: Unidad de Trauma Hospital Nacional de Niños. Act Méd Costarric 1999; 17-26.
14. Bianchini S, Espinoza D y Centeno C. Quemaduras infantiles y sus aspectos sociales. Revista Médica del Hospital Nacional de Niños Dr. Carlos Sáenz Herrera 1984, 19:107-111.

15. Wintemute G. Childhood drowning and near-drowning in the United States. Am J Dis Child 1990; 144: 663-669.

16. Quan L, Gore EJ, Wantz K. Ten year study of pediatric drowning and near-dorwning: lessons in injury prevention. Pediatrics 1989; 83: 1035-40.

17. Modena Hoover Wilson: Injury prevention and control. In: McMillan $\mathrm{J}$, DeAngelis $\mathrm{C}$ et al. Oski's Pediatrics: principles and practice. Third edition. Lippincot Williams \& Wilkins. Philadelphia; 1999. 491503.

18. Kohen D, Soubhi H and Porminder R. A canadian picture of maternal report of childhood injuries. British Columbia Research for Children and Women Health; 1999. 7-24.

19. Barlow, Niemirska M, Gandhi R. Ten years experience with falls from height in children. J Pediatr Surg 1983; 18: 509-11.

20. Bourguet $\mathrm{CC}, \mathrm{Mc}$ Artur RE. Unintentional injuries. Risk factors in preschool children. Am J Dis Child 1989; 143: 556-559.

21. Runyan CW. Risk factors for fatal residential fires. N Engl J Med 1992; 327: 859-863.

22. Kopjar B, Wickizer T. How safe are day care centers? Day care versus home injuries among children in Norway. Pediatrics 1996; 97: 43-47.

23. Cummings $\mathrm{P}$, Theis $\mathrm{MK}$, Mueller BA and Rivara F. Infant injury death en Washington State, 1981 through 1990. Arch Pediátr Adolesc Med 1994; 148: 1021-1026.

24. Wicklund K, Moss S, Frost F. Effects of maternal education, age and parity on fatal infant accidents. Am J Public Health 1984; 74: 11501152.

25. Nersesian W, Petit M, Shaper R, Lemieux D, and Naor E. Childhood death and poverty: a study of all childhood deaths in Maine, 1976 to 1980. Pediatrics 1988; 75: 41-50.

26. Scholer SJ, Hickson GB, Mitchel EF, Ray WA. Persistently increased injury mortality rates in high-risk young children. Arch Pediatr Adolesc Med 1997; 151: 1216-1219. 